\title{
Experiencing Organisational and Personal Change Management for Continuous Organisational Effectiveness
}

\author{
Jinasiri Dadallage ${ }^{1}$ \\ SLJDA 2015 (5): 27-36
}

\begin{abstract}
The paper argues how best change management can be implemented when using personal and organisational strategies. The paper shares the author's experiences in utilising best practices related to change management. It can be evident that managing organisational change will be more successful when applying simple principles i.e., thoughtful planning and sensitive implementation, and above all, consultation with, and involvement of, the people affected by the changes Also, achieving personal change will be more successful when used at relevant occasions. Change management cannot be implemented in a forceful environment and normally more problems will arise as a result. Change must be realistic, achievable and measurable. These aspects are especially relevant to managing personal change. Before starting organisational change, ask yourself: What do we want to achieve with this change, why, and how will we know that the change has been achieved? Who is affected by this change, and how will they react to it? How much of this change can we achieve ourselves, and with what parts of the change do we need help? The paper concludes with a description of how those personal and organisational change aspects become useful when realistically implementing change management.
\end{abstract}

Key words: Change Management, personal and organisational change, Sri Lanka

\section{Practice of Change}

I may argue that we do not have to promote change on people as a way of accelerating 'agreement' and implementation. Also "selling" change to people is not a sustainable strategy for success, unless your aim is to be

1 The author is the Secretary Ministry of Public Administration, Local Government and Democratic Governance. He joined to Sri Lanka Administrative Service (SLAS) in 1985 and during his career for more than 30 years he has served as a Head of Institutions, Departments and Ministries. Prior to his post as the Secretary, he served as the Director General of Sri Lanka Institute of Development Administration (SLIDA). The author holds a Bachelor of Education from University of Colombo and MSc in Human Resource Development from University of Manchester, United Kingdom. 
bitten at some time in the future when you least expect it. When people listen to top management 'selling' them change, diligent employees will generally smile and appear to accede, but quietly to themselves, they're thinking, "No chance mate, if you think I'm standing for that load of old rubbish you've another think coming..." Instead, change needs to be understood and managed in such a way that people can effectively cope with it. Therefore change can be unsettling, so the manager logically needs to be a settling influence.

It is important to check how people affected by the change agree with, or at least understand, the need for change, and have a chance to decide how the change will be managed, and to be involved in the planning and implementation of the change. Using face-to-face communications to handle sensitive aspects of organisational change management will convey meaning and understanding. In addition, encouraging managers to communicate face-to-face with employees when managing organisational change is important. I may claim that using emails and written notices are extremely weak communication approaches at conveying and developing inter personal understanding.

However, one best way of dealing with quick change is to probe the reasons - is the urgency real? Will the effects of agreeing on a more sensible time-frame really be more disastrous than presiding over a disastrous change? Quick change prevents proper consultation and involvement, which leads to difficulties that take time to resolve.

In addition, for complex changes, refer to the process of project management, and ensure that it is augmented with consultative communications for agreeing and gaining support for the reasons for the change. Involving and informing people also creates opportunities for others to participate in planning and implementing the changes, which lightens your burden, spreads the organisational load, and creates a sense of ownership and familiarity among the people affected. See also the excellent free decision making modal, designed by Sharon Drew Morgen (Morgen, 1997), with facilitative questions for personal and organisational innovation and change.

For organisational change there must be new actions, objectives and processes for a group or team of people, therefore use workshops or brainstorming sessions to achieve understanding, involvement, plans, measurable aims, actions and commitment. Also encourage the management team to use workshops with their teams when helping to manage the change. 
I may argue that there is a necessity to apply principles which are very difficult to change i.e., making people redundant, closures and integrating merged or acquired organisations. Bad news needs even more careful management than routine change. Hiding behind memos and middle managers will make matters worse. Consulting with people, and helping them to understand does not weaken your position - it strengthens it. Leaders who fail to consult and involve their employees in managing bad news are perceived as weak and lacking in integrity. Treat people with humanity and respect and they will reciprocate.

It is important to be mindful that the chief insecurity of most staff is change itself. Senior managers and directors responsible for managing organisational change do not, as a rule, fear change - they generally thrive on it. So remember that your staff do not relish change, they find it deeply disturbing and threatening. Your employees' fear of change is as great as your own fear of failure.

\section{Responsibility of managing change}

The employee does not have the responsibility to manage change - the employee's responsibility is no other than to do their best, which is different for every person and depends on a wide variety of factors i.e., health, maturity, stability, experience, personality, motivation and etc. Responsibility for managing change is with management and executives of the organisation - they must manage the change in a way that employees can cope with. The manager has the responsibility to facilitate and enable change, and all that is implied within that statement, especially to understand the situation from an objective standpoint (to 'step back', and be non-judgmental), and then to help people understand reasons, aims, and ways of responding positively according to employees' own situations and capabilities. Increasingly the manager's role is to interpret, communicate and enable - not to instruct and impose, which nobody really responds to well.

\section{People centric change}

What I meant by people centric change is to be cautious of expressions like 'mindset change', and 'changing people's mindsets' or 'changing attitudes'. Because the language often indicates a tendency towards imposed or enforced change, and it implies strongly that the organisation believes that its people currently have the 'wrong' mindset, which is never, ever, the case. If people are not approaching their tasks or the organisation effectively, then the organisation has the wrong mindset, 
not the people. In such an environment what may be important is to change towards new structures, policies, targets, acquisitions, disposals, re-locations, etc., all create new systems and environments, which need to be explained to people as early as possible, so that people's involvement in validating and refining the changes themselves can be obtained.

Whenever an organisation imposes new things on people there will be difficulties. Participation, involvement and open, early, full communication are the important factors. Workshops are very useful processes for developing collective understanding, approaches, policies, methods, systems, ideas, etc. Staff surveys are a helpful way to repair damage and mistrust among staff - provided you allow people to complete them anonymously, and provided you publish and act on the findings.

Management training, empathy and facilitative capability are priority areas - managers are crucial to the change process - they must enable and facilitate, not merely convey and implement policy from above, which does not work. Change should not be an "imposed change" people and teams need to be empowered to find their own solutions and responses, with facilitation and support from managers, and tolerance and compassion from the leaders and executives. Management and leadership style and behaviour are more important than clever processes and policy. Employees need to be able to trust the organisation. The leader must agree and work with these ideas, or change is likely to be very painful, and even the best people may be lost in the process.

There are some change management principles explained as follows.

1. At all times involve and agree on support from people within system (system $=$ environment, processes, culture, relationships, behaviours, etc., whether personal or organisational).

2. Understand where you/the organisation is at the moment.

3. Understand where you want to be, when, why, and what the measures will be for having got there.

4. Plan development towards No.3 above in appropriate achievable measurable stages.

5. Communicate, involve, enable and facilitate involvement from people, as early and openly and as fully as is possible. 


\section{Eight steps to successful change}

John Kotter's highly regarded books, 'Leading Change' (1995) and the follow-up 'The Heart of Change' (2002) describe a helpful model for understanding and managing change. Each stage acknowledges a key principle identified by Kotter relating to people's response and approach to change, in which people see, feel and then change Kotter's eight step change model can be summarised as:

1. Increase urgency - inspire people to move, make objectives real and relevant.

2. Build the guiding team - get the right people in place with the right emotional commitment, and the right mix of skills and levels.

3. Get the vision right - get the team to establish a simple vision and strategy and focus on emotional and creative aspects necessary to drive service and efficiency.

4. Communicate for buy-in - Involve as many people as possible, communicate the essentials simply, and appeal and respond to people's needs. De-clutter communications - make technology work for you rather than against.

5. Empower action - Remove obstacles, enable constructive feedback and lots of support from leaders - reward and recognise progress and achievements.

6. Create short-term wins - Set aims that are easy to achieve - in bitesize chunks. Manageable numbers of initiatives. Finish current stages before starting new ones.

7. Don't let up - Foster and encourage determination and persistence ongoing change - encourage ongoing progress reporting - highlight achieved and future milestones.

8. Make change stick - Reinforce the value of successful change via recruitment, promotion, and new change leaders. Weave change into the culture.

When people are confronted with the need or opportunity to change, especially when it is 'enforced', as perceived by the organisation, employees can become emotional. So can the managers who try to manage the change. To diffuse the emotional feelings, taking a step back and encouraging objectivity are important in enabling sensible and constructive dialogue. To this end, managers and trainers can find 
it helpful to use analogies to assist themselves and other staff to look at change in a more detached way. Aesop's fables have other short and beautifully simple analogies useful for illustrating aspects of causing or dealing with change, for example:

The Crow and the Pitcher (change being provoked by pressure or necessity)

The North Wind and the Sun (gentle persuasion rather than force)

The Lion and the Ass (enforced change - might is right)

The Crab and his Mother (lead by example and evidence - or you'll not change people)

The Miller, his Son and the Ass (no single change is likely to please everyone - everyone wants something different)

The Oak and the Reeds (the need for tolerance - changer or 'changees')

The Rich Man and the Tanner, (time softens change - given time people get used to things)

The Ass and the Mule (agree to reasonable change now or you can risk far worse enforced change in the future)

\section{Information technology and outsourcing for change}

There are various approaches to task analysis and job reorganisation, whether prompted by outsourcing or IT development. Generally a change process of this type is pragmatic, and it's difficult to identify transferable processes, templates, etc. These are some examples of projects which do not generally find their way into the public domain, although the likelihood of government project information being available on the web is increasing as such information is increasingly required to be available to the public. IT vendor case studies and trade journals of the IT and outsourcing sectors can also provide indicators of best practice or transferable processes. There are some useful software tools available, which are helpful, especially if the change involves a high level of complexity and is large in scale.

As a broad guide when managing this sort of change, these aspects are important for the process: 
- Correctly understand and clarify mutual expectations about the level of detail and cost that the project requires. Sometimes it's possible to see it what you need on a table napkin. The organisational context, and other strategic drivers, personalities and politics are often more significant influences than the task analysis.

- If you are a consultant or project manager, agree on expectations on a pragmatic basis. Agree on the templates and systems to be used and the level of report data required for the decision making.

- Assume that the situation can be improved - it generally can be, therefore while it's essential to capture all activities based on current jobs, many of these can be absorbed, superseded, updated, etc.

- A new overview analysis enables a fresh unencumbered look at the whole, which suggests new and better ways of doing things. A flip chart and a few creative minds are the main pre-requisites. It makes a great workshop session and is good for creating ownership and buy-in for major change. It is also a good process to cascade down to departments and bring out ideas for improved processes and new ways of doing things.

- In terms of capturing all current processes and inputs, the individual job analysis templates need to enable jobs to be broken down into sub-tasks, and elements within sub-tasks.

- Treat people at risk with the respect they deserve and avoid keeping them in the dark - involve threatened people wherever possible so that they can see what is happening and why. If possible encourage the executive team to take the same humane approach, and try to establish counselling and support resources if none exist already.

- Analyses are more helpful if they identify critical vs essential task elements - this will help you to help the decision-makers to be more pragmatic

- Flow diagrams identify subtask linkage (inter and intra), variation and chronology.

- Behaviour needs identifying, in addition to processes.

- Standards, performance tolerance, \% reliability, etc., should be indicated in task analysis as applicable to the sub-task or activity concerned. 


\section{Tips about people and change}

I would say that strong resistance to change is often rooted in deeply conditioned or historically reinforced feelings. Patience and tolerance are required to help people in these situations to see things differently. Bit by bit. There are examples of this sort of gradual staged change everywhere in the living world. Also, certain types of people - the reliable/dependable/steady/habitual/process-oriented types - often find change very unsettling.

People who welcomechange are not generally the best at being able to work reliably, dependably and follow processes. The reliability/dependability capabilities are directly opposite character traits to mobility/adaptability capabilities. Certain industries and disciplines have a high concentration of staff who need a strong reliability/dependability personality profile, for example, health services and nursing, administration, public sector and government departments, utilities and services. These sectors will tend to have many staff with character profiles who find change difficult.

Age is another factor. Erik Erikson's fascinating Psychosocial Theory (Erikson, 1981) is helpful in understanding that people's priorities and motivations are different depending on their stage of life. The more you understand people's needs, the better you will be able to manage change. Be mindful of people's strengths and weaknesses. Not everyone welcomes change. Take the time to understand the people you are dealing with, and how and why they feel like they do, before you take action.

\section{Fast changes needed in change management}

Planning, implementing and managing change in a fast-changing environment is increasingly the situation in which most organisations now work. Dynamic environments such as these require dynamic processes, people, systems and culture, especially for managing change successfully, notably effectively optimising organisational response to market opportunities and threats.

There are a few key elements for success as explained below,

- Plan long-term broadly - a sound strategic vision, not a specific detailed plan (the latter is impossible to predict reliably). Detailed five years plans are out of date two weeks after they are written. Focus on detail for establishing and measuring delivery of immediate actions, not medium-to-long-term plans. 
- Establish forums and communicating methods to enable immediate review and decision-making. Participation of interested people is essential. This enables their input to be gained, their approval and commitment to be secured, and automatically takes care of communicating the actions and expectations.

- Empower people to make decisions at a local operating level delegate responsibility and power as much as possible (or at least encourage people to make recommendations which can be quickly approved).

- Remove (as far as is possible) from strategic change and approval processes and teams (or circumvent) any ultra-cautious, ultraautocratic or compulsively-interfering executives. Autocracy and interference are the biggest obstacles to establishing a successful and sustainable dynamic culture and capability.

- Encourage, enable and develop capable people to be active in other areas of the organisation via 'virtual teams' and 'matrix management'.

- Scrutinise and optimise ICT (Information and Communications Technology) systems to enable effective information management and key activity team-working.

- Use workshops as a vehicle to review priorities, to agree on broad medium-to-long-term vision and aims, and to agree on short term action plans and implementation method and accountabilities.

- Adjust recruitment, training and development to accelerate the development of people who contribute positively to a culture of empowered dynamism.

\section{Conclusion}

Therefore it can be identified that the main issue is people's resistance and defensiveness towards someone coming in to their organisation to do what you are doing. When the manager overcomes that challenge, then he/she can start comparing what is happening with what the organisation sets out to do (mission, values, goals, priorities, targets, key performance indicators, processes, measures); how the people feel about things (staff turnover, retention, morale, attitudes); and how customers and suppliers feel about things (actually go out and visit customers, and ex-customers particularly). 
The manager needs to observe protocols very diligently - introduce him/ her properly to people and explain who you are and what you are doing. Don't assume that your task gives you the right to be secretive, or to have access to anyone or anything without permission. Ask for help. Ask for introductions. Ask for permission. Be polite and courteous. Respect people more than you would do normally, because they will be sensitive, which is understandable.

The manager must aim to help, enable and facilitate discovery and clarity, not work in splendid isolation as an outsider, who has come to 'sort things out', and then be lead by the people there on what can be improved. You should adopt the role of a researcher and enabler rather than a problem solver. Plan many questions that will help people to tell you how they feel - customers, staff and suppliers - and what they think can be done to improve things. Avoid asking 'why' unless they really trust you and are working with you. Used early, 'why' makes people defensive and you'll not find out anything. It's likely that you'll have to write a report and recommendations afterwards, in which case try wherever possible to involve the people in what you say about them. Let there be no surprises. Be constructive. Accentuate the positive. Be straight and open with people.

Enjoy the experience. Be respectful and helpful to people and they'll be respectful and helpful to you.

\section{References}

Erikson, E., \& Erikson, J. (1981). On generativity and identity: From a conversation with Erik and Joan Erikson. Harvard Educational Review, 51(2), 249-269.

Kotter, J. P., \& Cohen, D. S. (2002). The heart of change: Real-life stories of how people change their organizations. Harvard Business Press.

Morgen, S. D. (1997). Selling with integrity: Reinventing sales through collaboration, respect, and serving. Berrett-Koehler Publishers. 